\title{
The Highest-Derivative Version of Variational Perturbation Theory
}

\author{
B. Hamprecht and A. Pelster \\ Institut für Theoretische Physik, Freie Universität Berlin, \\ Arnimallee 14, D-14195 Berlin, Germany \\ E-mails: bodo.hamprecht@physik.fu-berlin.de, axel.pelster@physik.fu-berlin.de
}

\begin{abstract}
We systematically investigate different versions of variational perturbation theory by forcing not only the first or second but also higher derivatives of the approximant with respect to the variational parameter to vanish. The choice of the highest derivative version turns out to be the most successful one for approximating the ground-state energy of the anharmonic oscillator. It is therefore used to determine the critical exponent $\alpha$ of the specific heat in superfluid ${ }^{4} \mathrm{He}$ in agreement with the value measured in recent space shuttle experiments.
\end{abstract}

\section{INTRODUCTION}

The perturbative treatment of quantum-statistical or field-theoretical problems renders in general results in the form of divergent infinite power series in some coupling constant $g$. Typically, the coefficients of these series grow factorially with alternating signs leading to a zero convergence radius. Various resummation schemes may be applied to obtain finite results for all values of the coupling constant $g$, even in the strong-coupling limit $g \rightarrow \infty$ (for an overview see Chap. 16 of the book of Kleinert and Schulte-Frohlinde [1] and the references therein). Most successful is a recent systematic development by Kleinert [3], extending the variational method of Feynman and Kleinert [2] which was set up for calculating the effective classical potential in quantum statistics. It has been thoroughly tested for the ground-state energy of the anharmonic oscillator and shown to converge exponentially fast and uniform to the correct result 活. This was encouraging enough to apply the method also to divergent series which arise from renormalizing the ${ }^{4}$-theory of critical phenomena [1,5, 9, where the perturbation coefficients are available up to six and partly to seven loops in $d=3$ [10 12 and up to five loops in $d=4-\epsilon$ dimensions [13]. The method yielded finite results with a smooth dependence on the order. Furthermore, the theoretical results are in excellent agreement with the only experimental value available so far with an appropriate accuracy, the critical exponent $\alpha$ governing the behaviour of the specific heat near the superfluid phase transition of ${ }^{4} \mathrm{He} 11,15$.

Let us briefly recall the method. Consider some function $f(g)$ which is perturbatively obtained for a small coupling constant $g$ as the divergent weak-coupling series

$$
f(g)=\sum_{n=0}^{\infty} c_{n} g^{n}
$$

where the $c_{n}$ denote the respective expansion coefficients. Kleinert's variational perturbation theory [3] replaces the series (1.1) by

$$
f^{N}(g, p, q, w)=\left(\frac{g}{w}\right)^{p q} \sum_{n=0}^{N} c_{n} w^{n} \sum_{k=0}^{N-n}(-1)^{k}\left(\begin{array}{c}
p-\frac{n}{q} \\
k
\end{array}\right)\left(1-\left(\frac{w}{g}\right)^{q}\right)^{k} .
$$

By doing so, the newly introduced parameters $N, p, q, w$ are specified as follows. $N$ represents the order of the expansion and will be increased step by step as far as the knowledge of the weak-coupling coefficients $c_{n}$ permits. The parameters $p$ and $q$ determine the asymptotic behaviour of the function $f(g)$ in the strong-coupling limit $g \rightarrow \infty$ according to

$$
f(g)=g^{p q} \sum_{j=0}^{\infty} b_{j} g^{-j q},
$$

where the $b_{j}$ denote the strong-coupling coefficients. In quantum statistics the parameters $p$ and $q$ are usually known, e.g. from dimensional arguments, whereas in statistical field theory they are related to unknown critical exponents,

\footnotetext{
${ }^{1}$ In contrast to the standard notation 3 the parameter $q$ in Eq. (1.2) has been chosen in such a way that it coincides with the critical exponent $\Omega$ describing the approach to scaling in the field theory of critical phenomena [i].
} 
so they have to be determined self-consistently from the weak-coupling coefficients $c_{n}$ 䏤,6]. Finally, as the variational parameter $w$ is introduced such that the approximant (1.2) will not depend on it in the limit $N \rightarrow \infty$, it should be determined according to the principle of minimal sensitity [16. This principle is, however, no clear-cut mathematical statement and may therefore be implemented differently, giving rise to different versions of Kleinert's variational perturbation theory. Here we investigate and compare the versions, which define minimal sensitivity by the vanishing of a derivative of (1.2) with respect to $w$ to some order $k$. Such a version is considered to be good, if its results converge well to the true value in the quantum-statistical case, especially when $p$ is known, but $q$ is taken to be unknown, since this will anticipate the situation for the field-theoretical application. Another desirable feature can be seen in a very smooth dependence on the order $N$, since this will greatly enhance the possibility to extrapolate field-theoretical results to $N=\infty$. Finally, the simpler the version's defining prescription, the more easily it may be generalized to field-theoretical applications.

To begin with we focus our attention to the versions of variational perturbation theory in the strong-coupling limit $g \rightarrow \infty$. Comparing the Eqs. (1.2) and (1.3) gives, for the leading strong-coupling coefficient $b_{0}$, the expression

$$
b_{0}^{N}=(-1)^{N} w^{-p q} \sum_{n=0}^{N} c_{n}(-w)^{n}\left(\begin{array}{c}
p-1-\frac{n}{q} \\
N-n
\end{array}\right),
$$

where the variational parameter $w$ is still to be optimized. For the ground-state energy of the anharmonic oscillator potential

$$
V(x)=\frac{1}{2} x^{2}+g x^{4}
$$

the leading strong-coupling coefficient has the numerical value 17

$$
b_{0}=0.66798625915577710827096 \ldots
$$

and dimensional arguments require $q=2 / 3, p=1 / 2$. The weak-coupling coefficients $c_{n}$ are derived in the Appendix (see Tab. ฤ).

\section{THE HIGHEST-DERIVATIVE VERSION}

Traditionally, the expression (1.4) for $b_{0}$ is made stationary by forcing its first or second derivative with respect to the variational parameter $w$ to vanish, depending on whether the order $N$ is odd or even. Here we investigate whether some higher derivatives may be used instead. To this end we consider, for every order $N$, all derivatives $\partial^{k} b_{0}^{N} / \partial w^{k}$ with $k=1, \ldots, N+4$ and determine all real positive zeros $w$. For each of these zeros, the quantity $\log \left|b_{0}^{N}-b_{0}\right|$ measures the quality of approximating $b_{0}$ in Eq. (1.6) by $b_{0}^{N}$ in Eq. (1.4) (see Fig. 1).

Optimal results are obtained for $k=N$. Moreover, the prescription is unique, since for $k=N$ there exists only one real positive zero $w$ of $\partial^{k} b_{0}^{N} / \partial w^{k}$. It should be noted that for $k=N-1$ there never exists a real positive zero. Another unique and almost optimal prescription would be $k=N-2$ which works well for all $N>2$. The results for $k=1$ and $k=2$ are of comparable quality with the values for $k=N$.

Although only one of them has a real positive zero up to $N=5$, the equations for $k=1$ and $k=2$ tend to have more than one solution for larger $N$, the one with the largest value for $w$ always producing the best result. But there is no simple rule to decide whether $k=1$ or $k=2$ gives the better value. The initial indication of using $k=1$ for odd and $k=2$ for even orders does not carry through beyond $N=6$.

In Fig. 2 we compare the results for $k=N$ with the optimal branch chosen judiciously from the equations for $k=1$ and $k=2$. We also show a quadratic approximation to the $k=N-2$ equation where only the last three terms of the condition $\partial^{N-2} b_{0}^{N} / \partial w^{N-2}=0$ are kept, leaving us with a quadratic equation for the variational parameter $w$. It can be seen that this approximation is of high quality, as was to be expected.

This may be understood as follows: the coefficients of the $w$-expansion of $b_{0}^{N}$ in Eq. (1.4) alternate in sign and grow faster than $n$ !, which is the same for the weak-coupling coefficients $c_{n}$ from which the former are derived. Therefore the series (1.4), or any derivative thereof will preferably become zero, if neighbouring terms in the sum nearly cancel each other. The failure of such cancellation among some high order neighbours cannot easily be compensated by lower order terms because of the large order behaviour of the coefficients. Hence the vanishing of the derivative for $k=N-2$ can well be approximated with little loss of accuracy by choosing $w$ in such a way, that only the three highest order terms cancel each other. Thus we conclude that $k=N-2$ represents the highest non-trivial derivative version for applications in statistical field theory, where we usually have $p=0$, so the strong-coupling coefficient $b_{0}^{N}$ in Eq. (1.4) is a polynomial in $w$. It becomes the simpler the higher derivatives are being used. 

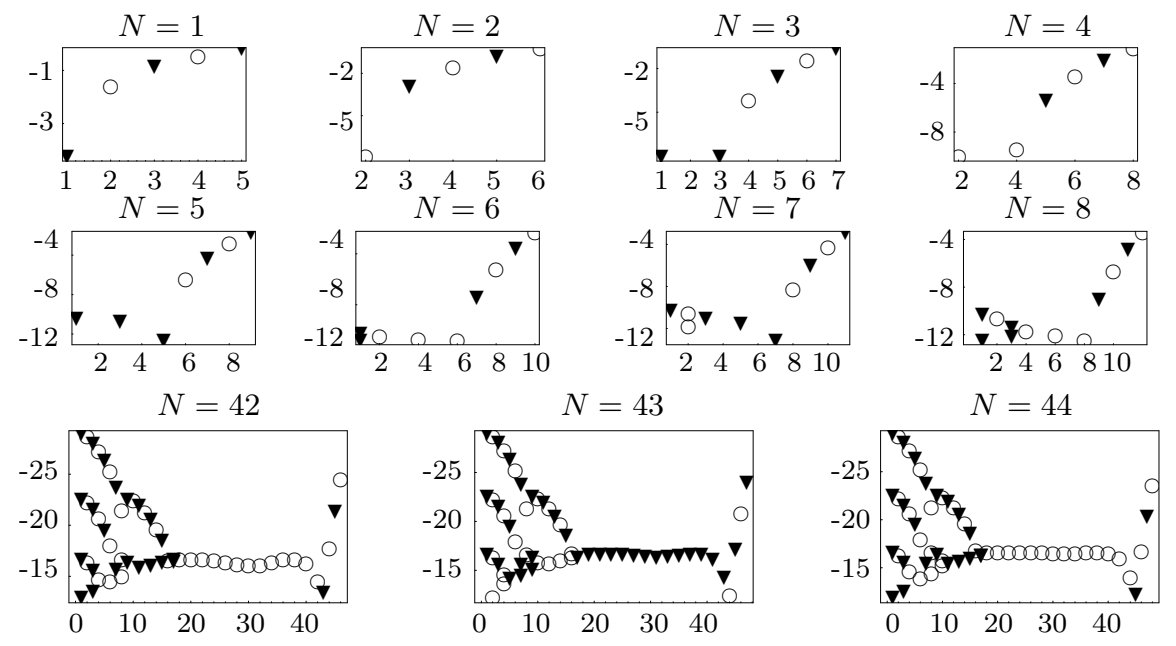

FIG. 1. The approach of $b_{0}^{N}$ to its true value $b_{0}$ is measured by the quantity $\log \left|b_{0}^{N}-b_{0}\right|$ which is plotted over $k$, where $k$ appears in the condition $\partial^{k} b_{0}^{N} / \partial w^{k}=0$ determining the variational parameter $w$. Some typical values for the order $N$ have been depicted with different symbols $\boldsymbol{\nabla}$ and $\circ$ used to plot odd and even $k$, respectively. Optimal results are obtained for $k=1$ or $k=2$, but with no simple rule as to which of the two has to be chosen. Taking $k=N$, however, the same quality is obtained with no ambiguity of choice. Also for $k=N-2$, the outcome is very reasonable. Note that for some of the lower values of $k$ there is more than one solution and that for some $k$, like e.g. for $k=N-1$, there is no solution at all.

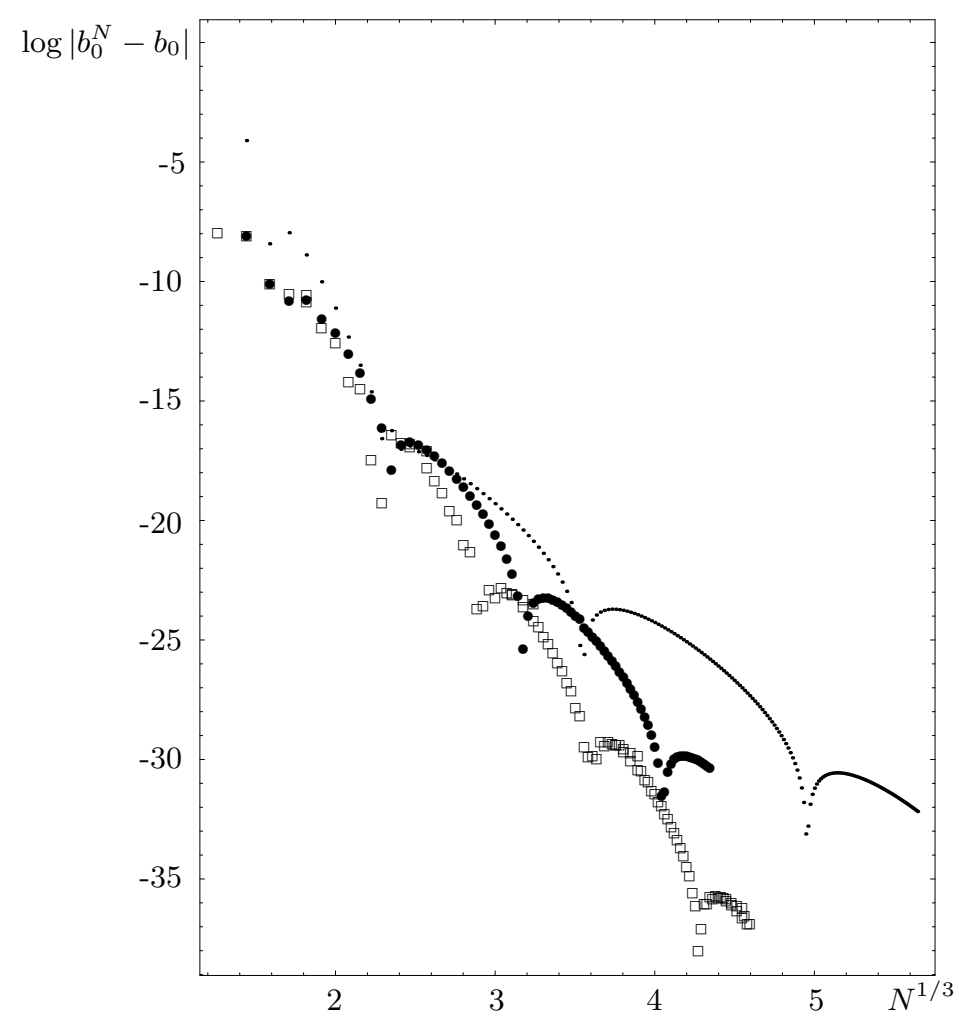

FIG. 2. The error $\log \left|b_{0}^{N}-b_{0}\right|$ plotted over $N^{1 / 3}$ as obtained by various methods: • for $k=N$, f for a quadratic approximation to $k=N-2$, and $\square$ for optimal values from $k=1$ or $k=2$, respectively. 


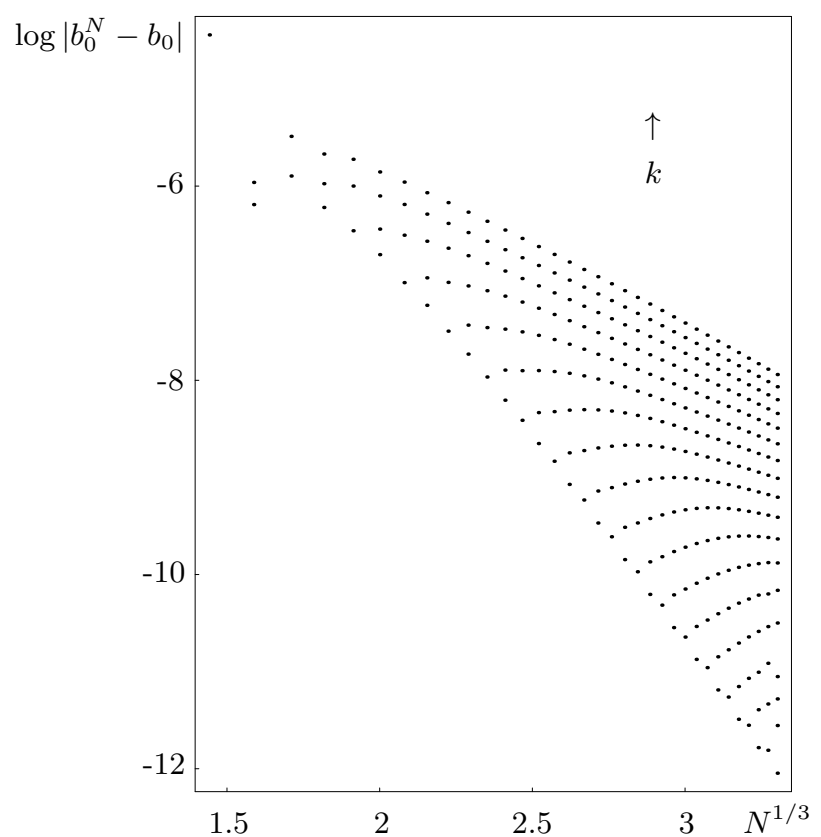

FIG. 3. For every $N$ several $q$-values are obtained by setting the $k$ th derivative of the strong-coupling limit of $F(g)=g f^{\prime}(g) / f(g)$ with respect to the variational parameter $w$ equal to zero. For each of these self-consistent values of $q$ the error $\log \left|b_{0}^{N}-b_{0}\right|$ is plotted over $N^{1 / 3}$. Smaller $k$ give better results, but the highest $k$-values up to $k=N-1$ are also tolerable.

\section{SELF-CONSISTENT DETERMINATION OF THE PARAMETER $Q$}

In this section we evaluate again the leading coefficient $b_{0}$ in the strong-coupling expansion for the ground-state energy of the anharmonic oscillator. But this time the parameter $q$ is not fixed to its proper value $q=2 / 3$. Instead we draw this information from the same weak-coupling coefficients $c_{n}$, whereas $p$ is set to its known value $p=1 / 2$. In order to determine $q$ we need an additional equation. This usually results from a self-consistent reasoning by evaluating the logarithmic derivative of the strong-coupling series (1.3) of $f(g)$ in the strong-coupling limit $g \rightarrow \infty$ [5, 6]:

$$
\lim _{g \rightarrow \infty} \frac{g f^{\prime}(g)}{f(g)}=\lim _{g \rightarrow \infty} \frac{g^{p q} \sum_{j=0}^{\infty} b_{j} q(p-j) g^{-j q}}{g^{p q} \sum_{j=0}^{\infty} b_{j} g^{-j q}}=p q=\frac{q}{2} .
$$

Defining $F(g):=g f^{\prime}(g) / f(g)$, the weak-coupling coefficients $\gamma_{n}$ of $F(g)$ are determined from the corresponding $c_{n}$ of $f(g)$ in Eq. (1.1). The strong-coupling limit of $F(g)$ can then be constructed with the help of variational perturbation theory by using $\gamma_{n}$ instead of $c_{n}$ in quite an analogous way as before. Since $F(g)$ tends to the constant value $q / 2$ in the strong-coupling limit $g \rightarrow \infty$, its parameter $p$ has to be zero, whereas $q$ must have the same value as before. Thus, from the Eqs. (1.4) and (3.1), we obtain for the strong-coupling limit of $F(g)$

$$
(-1)^{N} \sum_{n=0}^{N} \gamma_{n}(-w)^{n}\left(\begin{array}{c}
-1-\frac{n}{q} \\
N-n
\end{array}\right)=\frac{q}{2},
$$

where we determine the variational parameter $w$ on the left-hand side by demanding that the $k$ th derivative with respect to $w$ must vanish, $k$ being chosen appropriately. This set of equations can be rearranged into two polynomials of $q$ and $w$ which have to vanish simultaneously. The complete set of complex zeros becomes legion even for moderate orders $N$, their number growing with $N^{2}$. Constraining ourselves to real and positive solutions for $q$ in the expected range $0.5<q<0.9$, we find, in contrast to the previous case, a very regular behaviour. Exactly one unique solution exists for all $N$ up to $N=36$ and for all $k$ if $N+k$ is an odd integer, whereas there is no solution within this range if $N+k$ is even. The solutions for different $k$ approach the value $q=2 / 3$ closer for smaller $k$. As $k$ increases, the loss of accuracy, however, is tolerable, as can be seen from Fig. 3, where again the error $\log \left|b_{0}^{N}-b_{0}\right|$ is plotted over $N^{1 / 3}$, 
the approximation $b_{0}^{N}$ being obtained as before with the highest derivative version, but using the self-consistently determined $q$ instead of the exact $q=2 / 3$. Some loss of accuracy is the price to pay, if we want to make one of both equations linear by choosing $k=N-1$, which is now the highest non-trivial derivative. Notice that the penalty is relatively low for small orders $N$, which in field-theoretical models are the only ones available at present.

\section{CRITICAL EXPONENT $\alpha$ FOR LIQUID HELIUM}

After having analysed the highest derivative versions of variational perturbation theory by the example of the ground-state energy of the anharmonic oscillator, we apply this method to liquid ${ }^{4}$ He. In particular, we consider its superfluid state near the transition point $T_{c}$, in order to calculate the critical exponent $\alpha$ governing the power behaviour $\left|T_{c}-T\right|^{-\alpha}$ of the specific heat. Within the framework of the $\phi^{4}$-theory of critical phenomena, the superfluid phase transition of ${ }^{4} \mathrm{He}$ is described by a complex order parameter field $\phi$ whose bare energy functional is of the Ginzburg-Landau type and reads in $d=3$ dimensions [1]:

$$
E\left[\phi_{B}\right]=\int d^{3} x\left\{\frac{1}{2}\left[\partial \phi_{B}(x)\right]^{2}+\frac{1}{2} m_{B}^{2} \phi_{B}^{2}(x)+\frac{\pi}{5} g_{B}\left[\phi_{B}^{2}(x)\right]^{2}\right\} .
$$

By calculating the Feynman diagrams, one encounters divergencies which are removed by analytic regularization [18]. A subsequent renormalization leads to renormalized values of mass, coupling constant and field which are related to the bare input quantities by the respective renormalization constants $Z_{m}, Z_{g}$ and $Z_{\phi}$ :

$$
m_{B}^{2}=m^{2} Z_{m} Z_{\phi}^{-1}, \quad g_{B}=g Z_{g} Z_{\phi}^{-2}, \quad \phi_{B}=\phi Z_{\phi}^{1 / 2}
$$

In the literature, one finds expansions for these renormalization constants and for certain logarithmic derivatives, the so-called renormalization group functions, up to six and partly up so seven loops [10 12]. All these expansions can be written in terms of the dimensionless bare coupling constant $\bar{g}_{B}=g_{B} / \mathrm{m}$. For instance, one obtains, for the dimensionless renormalized coupling constant $\bar{g}=g / m$, the series

$$
\bar{g}\left(\bar{g}_{B}\right)=\sum_{n=0}^{\infty} \kappa_{n} \bar{g}_{B}^{n}
$$

The logarithmic derivative of the square mass ratio

$$
\eta_{m}\left(\bar{g}_{B}\right)=-\frac{d \log \frac{m^{2}}{m_{B}^{2}}}{d \log \bar{g}_{B}}
$$

reads correspondingly

$$
\eta_{m}\left(\bar{g}_{B}\right)=\sum_{n=0}^{\infty} \lambda_{n} \bar{g}_{B}^{n}
$$

Its weak-coupling coefficients $\kappa_{n}$ and $\lambda_{n}$ have been listed in Tab. I, respectively.

In the field-theoretic description of second-order phase transitions, the renormalized square mass $m^{2}$ vanishes near the transition point $T_{c}$. Thus the approach to scaling corresponds to the strong-coupling limit of the dimensionless

bare coupling constant $\bar{g}_{B}$ [1,5]. Expecting to obtain finite results for both (4.3) and (4.5), when the dimensionless bare coupling constant $\bar{g}_{B}$ tends to infinity, the strong-coupling expansions for $\bar{g}\left(\bar{g}_{B}\right)$ and $\eta_{m}\left(\bar{g}_{B}\right)$ should read

$$
\begin{array}{r}
\bar{g}\left(\bar{g}_{B}\right)=\bar{g}+\sum_{j=1}^{\infty} \beta_{j} \bar{g}_{B}^{-j \Omega}, \\
\eta_{m}\left(\bar{g}_{B}\right)=\eta_{m}+\sum_{j=1}^{\infty} \gamma_{j} \bar{g}_{B}^{-j \Omega} .
\end{array}
$$


TABLE I. Weak-coupling coefficients for $\bar{g}\left(\bar{g}_{B}\right)$ and $\eta_{m}\left(\bar{g}_{B}\right)$ from 6- and 7-loop perturbation theory 10 (above the tripple line) and from extrapolations based on instanton calculations [1, 18] (below the tripple line).

\begin{tabular}{|c|c|c|}
\hline \multicolumn{3}{|c|}{ Weak-Coupling Coefficients } \\
\hline & $\eta_{m}=\sum \lambda_{n} \bar{g}_{B}^{n}$ & $\bar{g}=\sum \kappa_{n} \bar{g}_{B}^{n}$ \\
\hline$n$ & $\lambda_{n}$ & $\kappa_{n}$ \\
\hline 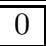 & 0 & 0 \\
\hline 1 & 4 & 1 \\
\hline 2 & -46.81481481481482 & -10 \\
\hline 3 & 667.3896935193186 & 120.1481481481481 \\
\hline 4 & -10792.61838744809 & -1642.256264617284 \\
\hline 5 & 191274.3323790051 & 24816.04561588786 \\
\hline 6 & -3644347.117315811 & -407363.539559348 \\
\hline 7 & 7378080.984900002 & \\
\hline$\overline{77}$ & & $7.180326000784143 \times E^{6}$ \\
\hline 8 & $-1.575312899817985 \times E^{9}$ & $-1.347981388551665 \times E^{8}$ \\
\hline 9 & $3.529433822947775 \times E^{10}$ & $2.679259494762891 \times E^{9}$ \\
\hline 10 & $-8.269004838427051 \times E^{I 1}$ & $-5.61279293532737 \times E^{10}$ \\
\hline 11 & $2.020940372700199 \times E^{13}$ & $1.234985211831956 \times E^{12}$ \\
\hline 12 & $-5.143391961273287 \times E^{14}$ & $-2.846297378479211 \times E^{13}$ \\
\hline 13 & $1.360628154286311 \times E^{16}$ & $6.837009909070767 \times E^{14}$ \\
\hline 14 & $-3.734409500947708 \times E^{17}$ & $-1.699763366416082 \times E^{16}$ \\
\hline 15 & $1.062320774475501 \times E^{19}$ & $4.35439267576015 \times E^{17}$ \\
\hline 16 & $-3.13267075119459 \times E^{20}$ & $-1.151470001163625 \times E^{19}$ \\
\hline 17 & $9.584565077380565 \times E^{21}$ & $3.166449929095059 \times E^{20}$ \\
\hline 18 & $-3.04462093970496 \times E^{23}$ & $-9.135728488698395 \times E^{21}$ \\
\hline 19 & $1.004119260969699 \times E^{25}$ & $2.780684275579437 \times E^{23}$ \\
\hline 20 & $-3.43537941718977 \times E^{26}$ & $-8.925916735892876 \times E^{24}$ \\
\hline 21 & $1.217685465986233 \times E^{28}$ & $3.006538451329012 \times E^{26}$ \\
\hline 22 & $-4.465282353357697 \times E^{29}$ & $-1.055624798462923 \times E^{28}$ \\
\hline 23 & $1.691830517012908 \times E^{31}$ & $3.841496075948586 \times E^{29}$ \\
\hline 24 & $-6.616032677503413 \times E^{32}$ & $-1.443458944165208 \times E^{31}$ \\
\hline
\end{tabular}

TABLE II. The critical exponent $\Omega$ as obtained from the strong-coupling limit of the first or the second logarithmic derivatives of the weak-coupling series for $\bar{g}\left(\bar{g}_{B}\right)$ and $\eta_{m}\left(\bar{g}_{B}\right)$, respectively.

\begin{tabular}{|c|c|c|c|c|}
\hline \hline \hline \multicolumn{5}{|c|}{$\Omega$-Values } \\
\hline$N$ & $\Omega(\bar{g}, 1)$ & $\Omega(\bar{g}, 2)$ & $\Omega\left(\eta_{m}, 1\right)$ & $\Omega\left(\eta_{m}, 2\right)$ \\
\hline \hline 2 & 0.730495 & 0.735397 & 0.715930 & 0.721303 \\
\hline 3 & 0.751627 & 0.751166 & 0.714270 & 0.712744 \\
\hline 4 & 0.757596 & 0.757762 & 0.703544 & 0.700880 \\
\hline 5 & 0.763865 & 0.763975 & 0.705086 & 0.704576 \\
\hline 6 & & & 0.714586 & 0.714540 \\
\hline \hline
\end{tabular}

TABLE III. The critical exponent $\alpha$ for the specific heat of liquid helium, derived from the perturbation expansion for $\eta_{m}$ using the highest-derivative version of variational perturbation theory.

\begin{tabular}{|c|c|c|}
\hline \hline \multicolumn{3}{|c|}{$\alpha$-Values } \\
\hline$N$ & $\eta_{m}$ & $\alpha$ \\
\hline \hline 2 & 0.490834 & +0.0121 \\
\hline 3 & 0.513786 & -0.0185 \\
\hline 4 & 0.522480 & -0.0304 \\
\hline 5 & 0.522651 & -0.0307 \\
\hline 6 & 0.519592 & -0.0265 \\
\hline \hline
\end{tabular}


Note that strong-coupling expansions like (4.6) and (4.7) are governed by one and the same critical exponent $\Omega$ [1], a common feature of second-order phase transitions. Thus from either of these series $\Omega$ may be extracted in various ways, taking logarithmic derivatives with respect to the dimensionless bare coupling constant $\bar{g}_{B}$. The strong-coupling behaviour (4.6) of the dimensionless renormalized coupling constant $\bar{g}\left(\bar{g}_{B}\right)$ implies, for instance,

$$
\begin{aligned}
\lim _{\bar{g}_{B} \rightarrow \infty} \frac{d \log \bar{g}\left(\bar{g}_{B}\right)}{d \log \bar{g}_{B}} & =0, \\
\lim _{\bar{g}_{B} \rightarrow \infty} \frac{d \log \bar{g}^{\prime}\left(\bar{g}_{B}\right)}{d \log \bar{g}_{B}} & =-\Omega-1,
\end{aligned}
$$

with corresponding results for $\eta_{m}\left(\bar{g}_{B}\right)$, as can easily been seen from Eq. 4.7). Forcing these relations upon the weak-coupling expansions as well, we obtain equations to which the strong-coupling limit of variational perturbation theory can be applied. We use its highest derivative version as explained above and find good agreement between the $\Omega_{N}$-values from each of the four equations (see Tab. [I]).

In fact they are supposed to approach the same limit as $N \rightarrow \infty$. We notice, that the members of each pair obtained from either the $\eta_{m}$ - or the $\bar{g}$ - expansion are almost the same, whereas the different pairs have not yet converged so well for $N \leq 6$. Even though the data from the $\bar{g}$-expansion look more promising because of their smoother behaviour, we still use the results $\Omega_{N}$ from the $\eta_{m}$-expansion alone to calculate the critical exponent $\alpha$, and do not use any information from the $\bar{g}$-expansion at this stage. We evaluate the critical exponent $\eta_{m}$ from

$$
\eta_{m}^{N}=(-1)^{N} \sum_{n=0}^{N} \lambda_{n}\left(-w_{N}\right)^{n}\left(\begin{array}{c}
-1-\frac{n}{\Omega_{N}} \\
N-n
\end{array}\right),
$$

with the variational parameter $w_{N}$ fixed by

$$
w_{N}=-\frac{\lambda_{N-1}\left(N-1+\Omega_{N}\right)}{\lambda_{N} N \Omega_{N}} .
$$

Here the $\Omega_{N}$ are supplied from the $\Omega\left(\eta_{m}, 1\right)$-column of Tab. II. The corresponding results are shown in Tab. [II along with the corresponding values for the critical exponent $\alpha$ of the specific heat which follows from

$$
\alpha=\frac{1-2 \eta_{m}}{\eta_{m}-2} .
$$

These are values quite close to the experimental data $\alpha_{\exp }=-0.01056 \pm 0.00038$ [15], thus giving support for the method used.

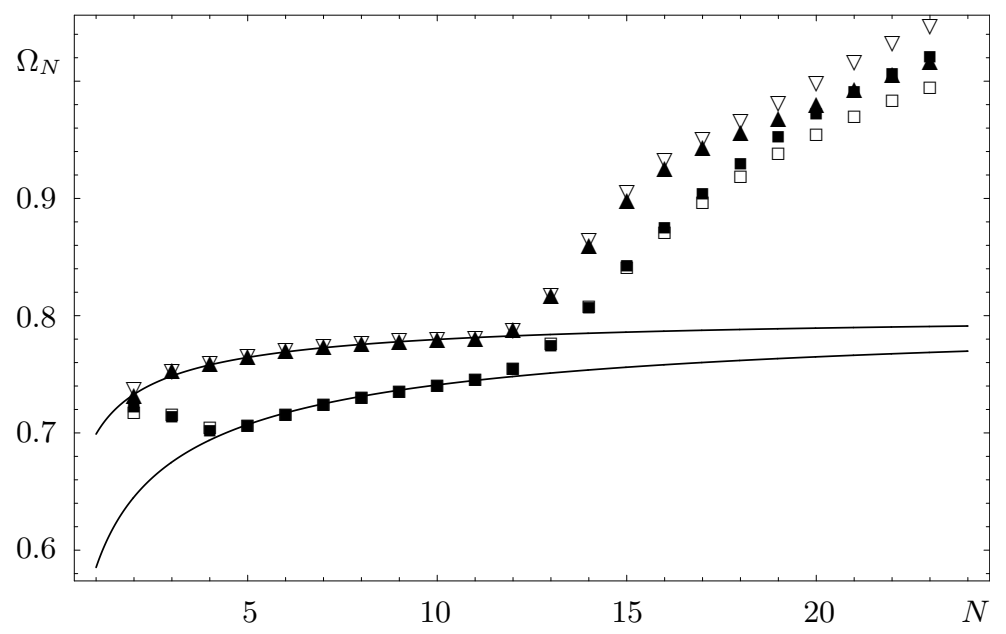

FIG. 4. The critical exponent $\Omega$ derived from the strong-coupling limit of the first and the second logarithmic derivative of the weak-coupling expansion of $\bar{g}\left(\bar{g}_{B}\right)(\boldsymbol{\Lambda}, \nabla)$ and of $\eta_{m}\left(\bar{g}_{B}\right)(\boldsymbol{\square}, \square)$, respectively. The solid lines are extrapolations with $\Omega_{\infty}=0.8$ fitted to the $\bar{g}$ - and $\eta_{m}$-points in the reliable interval $3<N<12$, respectively. 


\section{IMPROVEMENT BY EXTRAPOLATION}

Higher order perturbation coefficients for $\bar{g}\left(\bar{g}_{B}\right)$ and $\eta_{m}\left(\bar{g}_{B}\right)$ are known approximately (see Tab. I). They have been derived from instanton calculations, which were fitted to the weak-coupling data [7, 18]. Extending the previous calculation on this basis up to the order of $N=24$, good convergence of all four different sets of the $\Omega_{N}$ is found. We notice, however, a kink at about $N=12$ in Fig. A, which strongly suggests, that the extrapolation is no longer reliable beyond this point. For large $N$ the results $\Omega_{N}$ have the asymptotic form [1,5] 9]

$$
\Omega_{N}=\Omega_{\infty}-a \exp \left(-b N^{1-\Omega_{\infty}}\right)
$$

where the constants $a$ and $b$ are different for the $\eta_{m^{-}}$and the $\bar{g}$ - expansion. Unfortunately, the fit is extremely insensitive to the value of $\Omega_{\infty}$ : all values in a broad interval around $\Omega_{\infty}=0.8$ would fit the data up to $N=12$ from both series with very much the same quality (see Fig. 田). In Fig. 5 the resulting $\alpha$-values are plotted against the asymptotic form

$$
\alpha_{N}=\alpha_{\infty}-c \exp \left(-d N^{1-\Omega_{\infty}}\right)
$$

for $\alpha_{\infty}=-0.0106$ and $\Omega_{\infty}=0.8$. Up to the kink at $N=12$, the data seem to approach the experimental number excellently.

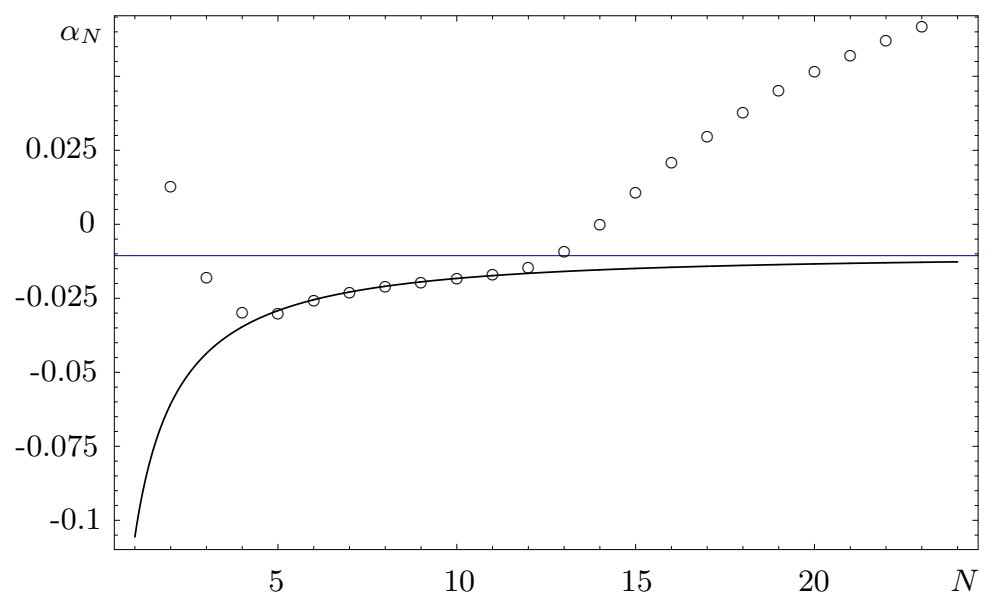

FIG. 5. The critical exponent $\alpha$ of the specific heat of liquid ${ }^{4} \mathrm{He}$ near the superfluid phase transition plotted against the $\operatorname{order} N$. The straight horizontal line represents the experimental value of $\alpha_{\exp }=-0.0106$. The curved line is an extrapolation of the results from the range of $3<N<12$.

TABLE IV. Comparison of quality for the highest-derivative version and for the traditional method, based on convergence of the critical exponent $\Omega$ obtained in two different ways from two different sets of data.

\begin{tabular}{|c|c|r|r|r|}
\hline \hline \multicolumn{5}{|c|}{$\sigma_{\Omega}$} \\
\hline data & method & $2 \leq N \leq 6$ & $7 \leq N \leq 12$ & $13 \leq N \leq 24$ \\
\hline \hline $\bar{g}\left(\bar{g}_{B}\right)$ & high & 0.002159 & 0.000570 & 0.017682 \\
\hline $\bar{g}\left(\bar{g}_{B}\right)$ & low & 0.002245 & 0.000522 & 0.000274 \\
\hline \hline$\eta_{m}\left(\bar{g}_{B}\right)$ & high & 0.002749 & 0.000258 & 0.016251 \\
\hline$\eta_{m}\left(\bar{g}_{B}\right)$ & low & 0.008214 & 0.008121 & 0.005915 \\
\hline \hline both & high & 0.046663 & 0.040836 & 0.037912 \\
\hline both & low & 0.046549 & 0.057049 & 0.055477 \\
\hline \hline
\end{tabular}




\section{QUALITY CHECK FOR THE HIGHEST DERIVATIVE VERSION}

The four different methods, discussed before to extract the critical exponent $\Omega$ from the data, should all give results which converge to the same limit as $N \rightarrow \infty$. We show now that the highest derivative version reveals a better convergence than the traditional method which uses the first or second derivatives instead. The details are reported in Tab. IV, where the variance $\sigma_{\Omega}$ between the values $\Omega_{N}^{(1)}, \Omega_{N}^{(2)}$ from different methods is listed for specific ranges of $N$ :

$$
\sigma_{\Omega}=\sqrt{\frac{1}{N_{1}-N_{0}+1} \sum_{N=N_{0}}^{N_{1}}\left(\Omega_{N}^{(1)}-\Omega_{N}^{(2)}\right)^{2}} .
$$

In the first row of Tab. IV we list the $\Omega$-values extracted from the weak-coupling expansion $(4.3)$ of $\bar{g}\left(\bar{g}_{B}\right)$ according to the methods (4.8), (4.9) using the highest derivative version. The same quantity evaluated in the traditional way, i.e. using the first derivative for even $N$ and the second derivative for odd $N$, is shown, for comparison, in the second row. It can be seen that both methods are of the same quality in the range of $2 \leq N \leq 6$, for which the calculation is based on the weak-coupling coefficients from perturbation theory, and also in the range of $7 \leq N \leq 12$, based on extrapolated values. Only for $N>12$, where the extrapolation is no more reliable, anyhow, the highest derivative version seems to be worse than the one obtained by the traditional method. However, in order to obtain these apparently better results using low order derivatives, the rules of the game had to be changed somewhat, taking the second derivative throughout for even and odd order $N$, as soon as $N$ becomes larger than 12 .

The next two rows show the corresponding comparison based on the weak-coupling expansion $(4.5)$ of $\eta_{m}\left(\bar{g}_{B}\right)$. Here it turns out that the highest derivative version gives results of significantly better quality than the traditional method, up to $N=12$.

Finally, in the last two rows the deviations of the $\Omega$-values from the weak-coupling expansions $(4.3)$ of $\bar{g}\left(\bar{g}_{B}\right)$ and (4.5) of $\eta_{m}\left(\bar{g}_{B}\right)$, extracted according to (4.8) are shown in comparison for the highest derivative version (above) and the traditional method (below). It can be seen, that both methods are more or less comparable in quality, the highest derivative version giving slightly better results.

\section{APPENDIX}

The perturbative solution of the time-independent Schrdinger equation

$$
H \Psi_{m}:=\left(H_{0}+g V\right) \Psi_{m}=E_{m} \Psi_{m}
$$

usually leads to infinite sums for the expansion coefficients of the energy eigenvalues $E_{m}(g)$ and the state vectors $\Psi_{m}(g)$. But if the perturbing potential $V$ happens to be band-diagonal in the basis of the eigenvectors of the unperturbed Hamiltonian $H_{0}$, then all these sums become finite and the eigenvalues $E_{m}(g)$ can be determined recursively to all orders.

Here we assume that the spectrum $\epsilon_{0}^{(0)}, \epsilon_{0}^{(1)}, \epsilon_{0}^{(2)}, \ldots, \epsilon_{0}^{(m)}, \ldots$ of the unperturbed Hamiltonian $H_{0}$ is non-degenerate and denote the corresponding eigenvectors by $|0\rangle,|1\rangle,|2\rangle, \ldots,|m\rangle, \ldots$ Following the usual procedure, the energy eigenvalues $E_{m}(g)$ and the state vectors $\Psi_{m}(g)$ are expanded in powers of $g$ :

$$
\begin{aligned}
& \Psi_{m}(g)=\sum_{n, i} \gamma_{n, i}^{(m)} g^{i} \alpha_{n}|n\rangle, \\
& E_{m}(g)=\sum_{j} \epsilon_{j}^{(m)} g^{j},
\end{aligned}
$$

where the $\alpha_{n}$ are inserted into the definition, for later computational convenience. They will only show up in the coefficients of the state vector $\Psi_{m}$, but not in the expansion of the energy eigenvalues $E_{m}$ which we are looking for. Without loss of generality the normalisation of the state vectors $\Psi_{m}$ is chosen such that $\left\langle\Psi_{m} \mid m\right\rangle=\alpha_{m}$ to all orders so that we have

$$
\gamma_{m, i}^{(m)}=\delta_{i, 0} \quad \gamma_{k, 0}^{(m)}=\delta_{k, m}
$$

Inserting the ansatz (7.2), (7.3) into the Schrdinger equation (7.1), projecting the result onto the base vector $\langle k| \alpha_{k}$ and extracting the coefficient of $g^{j}$, we obtain the relation: 


$$
\gamma_{k, i}^{(m)} \epsilon_{0}^{(k)}+\sum_{n} \frac{\alpha_{n}}{\alpha_{k}} V_{k, n} \gamma_{n, i-1}^{(m)}=\sum_{j} \epsilon_{j}^{(m)} \gamma_{k, i-j}^{(m)}
$$

with the matrix elements

$$
V_{k, n}:=\langle k|V| n\rangle .
$$

For $i=0$ this equation is identically satisfied. For $i \neq 0$ we get the following two relations, one for $k=m$ and the other one for $k \neq m$ :

$$
\begin{aligned}
\epsilon_{i}^{(m)} & =\sum_{n} \gamma_{m+n, i-1}^{(m)} W_{m, n} \\
\gamma_{k, i}^{(m)} & =\frac{1}{\epsilon_{0}^{(k)}-\epsilon_{0}^{(m)}}\left[\sum_{j=1}^{i-1} \epsilon_{j}^{(m)} \gamma_{k, i-j}^{(m)}-\sum_{n} \gamma_{k+n, i-1}^{(m)} W_{k, n}\right] .
\end{aligned}
$$

In these expressions all sums are finite if the potential $V$ is band-diagonal such that the augmented matrix elements

$$
W_{m, n}:=V_{m, m+n} \frac{\alpha_{m+n}}{\alpha_{m}}
$$

are different from zero only for $-d<n<d$, where $d$ is some finite number. For any quantum number $m$ these relations may now be applied recursively with respect to the order, leading to $\epsilon_{1}^{(m)}, \gamma_{k, 1}^{(m)}, \epsilon_{2}^{(m)}, \gamma_{k, 2}^{(m)}, \ldots, \epsilon_{r}^{(m)}, \gamma_{k, r}^{(m)}, \ldots$ in turn. Notice that for given $m$ and $r$ only a finite number of the $\gamma_{k, r}^{(m)}$ is non-zero.

Considering in particular the anharmonic oscillator with the unperturbed Hamiltonian $H_{0}=\left(p^{2}+x^{2}\right) / 2$ and the perturbing potential $V=x^{4}$, we may choose $\alpha_{k}=\sqrt{2^{k} / k !}$ to get the non-vanishing augmented matrix elements from (7.6) and (7.9). An easy way to calculate the augmented matrix elements starts from the ones for $V=x$, where we have

$$
\begin{gathered}
W_{k,-1}^{x}=k / 2 \\
W_{k, 1}^{x}=1 .
\end{gathered}
$$

From here the augmented matrix elements $W_{k, j}^{x^{l}}$ for any polynomial $V=x^{l}$ can be determined recursively by applying the rules of matrix multiplication:

$$
W_{k, j}^{x^{l}}=\sum_{i} W_{k, i}^{x} W_{k+i, j-i}^{x^{l-1}}
$$

Thus we obtain for $V=x^{4}$ :

$$
\begin{aligned}
W_{k,-4}^{x^{4}} & =k(k-1)(k-2)(k-3) / 16, \\
W_{k,-2}^{x^{4}} & =k(2 k-1)(k-1) / 4, \\
W_{k, 0}^{x^{4}} & =3\left(2 k^{2}+2 k+1\right) / 4, \\
W_{k, 2}^{x^{4}} & =2 k+3 \\
W_{k, 4}^{x^{4}} & =1 .
\end{aligned}
$$

Being rational numbers they are suitable for recursive calculations even up to very high orders. In Tab. $\mathrm{V}$ we have listed the first ten coefficients $\epsilon_{k}^{(0)}$ for the expansion of the ground-state energy $E_{0}(g)$. 
TABLE V. The first ten coefficients $\epsilon_{k}^{(0)}$ of the weak-coupling expansion for the ground-state energy $E_{0}(g)$ of the anharmonic oscillator.

\begin{tabular}{|c|c|}
\hline \hline \multicolumn{2}{|c|}{ The Anharmonic Oscillator } \\
\hline$k$ & $\epsilon_{k}^{(0)}$ \\
\hline \hline 0 & $1 / 2$ \\
\hline 1 & $3 / 4$ \\
\hline 2 & $-21 / 8$ \\
\hline 3 & $333 / 16$ \\
\hline 4 & $-30885 / 128$ \\
\hline 5 & $916731 / 256$ \\
\hline 6 & $-65518401 / 1024$ \\
\hline 7 & $2723294673 / 2048$ \\
\hline 8 & $-1030495099053 / 32768$ \\
\hline 9 & $54626982511455 / 65536$ \\
\hline 10 & $-6417007431590595 / 262144$ \\
\hline \hline
\end{tabular}

\section{ACKNOWLEDGEMENT}

It is a particular pleasure for B.H. to contribute to this volume in honor of Hagen Kleinert, looking back gratefully on four decades of friendship originating in the physics undergraduate courses of Technische Universität Hannover, incorporating a common year at Boulder University as well as the last 32 years as colleagues together at the Freie Universität Berlin. This is written with great admiration for Hagen's deep dedication to the miracles of nature and their physical interpretation.

[1] H. Kleinert and V. Schulte-Frohlinde, Critical Properties of $\phi^{4}$-Theories (World Scientific, Singapore, 2001).

[2] R.P. Feynman and H. Kleinert, Phys. Rev. A 34, 5080 (1986).

[3] H. Kleinert, Path Integrals in Quantum Mechanics, Statistics and Polymer Physics, 2nd edition (World Scientific, Singapore, 1995).

[4] W. Janke and H. Kleinert, Phys. Rev. Lett. 75, 2787 (1995); H. Kleinert and W. Janke, Phys. Lett. A 206, 283 (1995).

[5] H. Kleinert, Phys. Rev. D 57, 2264 (1998); Addendum, Phys. Rev. D, 58, 107702 (1998).

[6] H. Kleinert, Phys. Lett. B 434, 74 (1998).

[7] H. Kleinert, Phys. Rev. D 60, 085001 (1999).

[8] H. Kleinert Phys. Lett. B 463, 69 (1999).

[9] H. Kleinert and V. Schulte-Frohlinde, J. Phys. A 34, 1037 (2001).

[10] B.G. Nickel, D.I. Meiron, and G.B. Baker Jr., University of Guelph, preprint (1977), http://www.physik.fu-berlin.de/ ${ }^{\sim}$ kleinert/kleiner_reb8/programs/programs.html.

[11] S.A. Antonenko and A.I. Sokolov, Phys. Rev. E 51, 1894 (1995).

[12] D.B. Murray and B.G. Nickel, University of Guelph, preprint (1998).

[13] H. Kleinert, J. Neu, V. Schulte-Frohlinde, K.G. Chetyrkin, and S.A. Larin, Phys. Lett. B 272, 39 (1991); Phys. Lett. B 319, 545(E) (1993).

[14] H. Kleinert, Phys. Lett. A 277, 205 (2000).

[15] J.A. Lipa, D.R. Swanson, J.A. Nissen, T.C.P. Chui, and U.E. Israelsson, Phys. Rev. Lett. 76, 944 (1996); J.A. Lipa, D.R. Swanson, J.A. Nissen, Z.K. Geng, P.R. Williamson, D.A. Stricker, T.C.P. Chui, U.E. Israelsson, and M. Larson, Phys. Rev. Lett. 84, 4894 (2000). In the second paper, the value for the critical exponent of the specific heat of supfluid ${ }^{4}$ He given in the first paper was corrected to $\alpha_{\exp }=-0.01056 \pm 0.0004$.

[16] P.M. Stevenson, Phys. Rev. D 23, 2916 (1981).

[17] F. Vinette and J. Č́̌žek, J. Math. Phys. 32, 3392 (1991).

[18] G. Parisi, J. Stat. Phys. 23, 49 (1980). 